\title{
Pannes et accidents, mises en question et révélateurs des relations entre techniques, économie et société $\left(\mathrm{XIX}^{\mathrm{e}}-\mathrm{XXI}^{\mathrm{e}}\right.$ siècle)
}

\section{Guy Lambert et Olivier Raveux}

\section{(2) OpenEdition}

\section{Édition électronique}

URL : http://journals.openedition.org/artefact/4432

DOI : 10.4000/artefact.4432

ISSN : 2606-9245

Éditeur :

Association Artefact. Techniques histoire et sciences humaines, Presses universitaires du Midi

\section{Édition imprimée}

Date de publication : 15 décembre 2019

Pagination : 9-17

ISBN : 978-2-8107-0667-9

ISSN : 2273-0753

\section{Référence électronique}

Guy Lambert et Olivier Raveux, "Pannes et accidents, mises en question et révélateurs des relations entre techniques, économie et société (xıx $-\mathrm{xxl}^{\mathrm{e}}$ siècle) », Artefact [En ligne], 11 | 2019, mis en ligne le 27 novembre 2020, consulté le 29 novembre 2020. URL : http://journals.openedition.org/artefact/4432 ; DOI : https://doi.org/10.4000/artefact.4432

\section{(c) (i) (9)}

Artefact, Techniques, histoire et sciences humaines est mise à disposition selon les termes de la Licence Creative Commons Attribution - Pas d'Utilisation Commerciale - Pas de Modification 4.0 International. 


\section{Pannes et accidents, mises en question et révélateurs des relations entre techniques, économie et société $\left(\mathrm{XIX}^{\mathrm{e}}-\mathrm{XXI}^{\mathrm{e}}\right.$ siècle)}

\section{Guy Lambert et Olivier Raveux}

Ce dossier est dédié à la mémoire de notre collègue Larissa Zakharova, qui avait accueilli avec enthousiasme l'invitation à y prendre part.

$\mathrm{E}$ n dehors de travaux concernant les conditions de travail, le risque industriel ou la question environnementale ${ }^{1}$, les pannes et les accidents des systèmes de production de biens et de services n'ont pas encore retenu l'attention suffisante des historiens de l'économie, du social et des techniques de l'époque contemporaine. Le fait peut paraitre surprenant de prime abord. Les pannes et les accidents ne sont en effet pas des faits rares ou incongrus, mais rythment bien au contraire la marche et le

1. Il est impossible de livrer une bibliographie, même réduite, de ce thème de recherche et nous nous limitons donc à donner quelques exemples emblématiques de ces travaux : Bronstein, 2008 ; Fressoz, 2012 ; Le Roux (dir.), 2016 ; Lekéal, Dubois, Crochepeyre (dir.), 2016.

99 Guy Lambert et Olivier Raveux, «Pannes et accidents, mises en question et révélateurs des relations entre techniques, économie et société ( $\mathrm{XIX} \mathrm{X}^{\mathrm{e}}-\mathrm{XXI} \mathrm{e}^{\mathrm{e}}$ siècle) », Artefact, 11, 2019, p. 9-17. 
quotidien des systèmes techniques ${ }^{2}$. Pouvant être définis de manière large comme des arrêts soudains - involontaires ou non - du fonctionnement d'appareils, de machines ou de dispositifs pour les premières, et comme incidents inattendus entraînant des dommages sur les personnes et les biens pour les seconds, ces deux types d'aléas constituent des crises génératrices d'une documentation aussi abondante que riche qualitativement. Sources d'archives et dossiers de presse permettent en effet d'étudier, par un angle d'attaque décalé, des éléments placés au cœur du fonctionnement des systèmes techniques, des économies et des sociétés.

\section{Un observatoire du quotidien des techniques}

Avec les pannes et les accidents, le chercheur dispose d'un observatoire heuristique de tout premier ordre pour observer le quotidien des techniques ${ }^{3}$. Outre que ces sujets d'études offrent notamment la possibilité d'étudier la question de la maintenance préventive et curative des machines, de l'outillage et des dispositifs, ils invitent à porter la focale sur les acteurs qui en ont la charge. Le bon fonctionnement d'un procédé ou d'un appareil peut donner l'illusion que les techniques se suffisent à elles-mêmes pour être opérationnelles et performantes. L'étude des incidents de production de biens ou des services permet la réintroduction de l'humain dans la marche des techniques, comme le montre la contribution de Guillaume Carnino et de Clément Marquet sur l'histoire des pannes des datacenters. Ce travail fait apparaître les "petits » acteurs de la société d'information, ceux qui ont été rendus invisibles par la focalisation de la recherche sur les grands noms de l'innovation dans le secteur de l'informatique. Ces "petites mains", douées des savoir-faire traditionnels des métiers techniques, se retrouvent même en première ligne dans la gestion quotidienne et récurrente des incidents et dans la conservation de la maittrise opératoire des dispositifs techniques. Ce constat pourrait faire écho aux réalités de la mécanisation des outillages de chantier à la fin du XIX ${ }^{\mathrm{e}}$ siècle qu'aborde Guy Lambert à travers l'exemple des fondations par air comprimé. Généralement célébré pour sa

2. David Landes l'a rappelé pour tous les secteurs de la première révolution industrielle (Landes, 1975, p. 125), mais ce constat peut être perçu comme " universel ». Sur ce point, on lira également, Chynoweth, 2009.

3. Charpy, Jarrige, 2012. 
rapidité d'exécution et son efficacité que rend possibles l'application de ce mode d'énergie industriel au génie civil, ce procédé apparaît ici tributaire d'une main-d'œuvre, certes déqualifiée, mais dont la résistance physique est requise par les conditions mêmes de ce travail.

Porter attention aux pannes et aux accidents permet également de saisir et de questionner les avantages et les limites d'une technologie, de déconstruire un système technique et ses composantes, de réfléchir sur les choix effectués par les acteurs de l'économie dans le domaine des techniques. En étudiant le débat sur le choix de la vapeur ou de la force animale dans la mécanisation du battage des grains dans les campagnes françaises du $\mathrm{xIX}^{\mathrm{e}}$ siècle, François Jarrige révèle comment les accidents ont modelé les choix techniques des producteurs, des ingénieurs et des pouvoirs publics. De même, à travers l'étude d'un accident provoqué par un ascenseur dans le Paris de 1900, Manuel Charpy montre comment l'événement survient dans une phase de transition entre le système hydraulique et le règne du tout électrique. La documentation rassemblée autour de l'incident et du décès d'un usager livre le mode d'emploi du dispositif technique qui s'appuie sur une combinaison de nouveautés et d'éléments éprouvés par le temps.

L'intérêt que le chercheur peut retirer d'une enquête sur ces objets peut également s'observer sur le terrain de la technologie, entendue comme science de la pratique, à un moment où celle-ci a été éprouvée, amenant des réflexions autour des causes des pannes et des accidents, sur la vulnérabilité aux éléments extérieurs, sur les défauts de fabrication et de conception, sur les manquements dans les opérations de maintenance ou de conduite des machines ou des dispositifs techniques 4 . "De même que les médecins considèrent la santé comme la norme et la maladie comme l'exception, et qu'ils tirent la plus grande partie de leurs connaissances des maladies elles-mêmes, les ingénieurs considèrent les constructions qui tiennent debout comme la norme et celles qui s'effondrent comme l'exception, apprenant tout autant de ces échecs 5 . " Il s'agit alors d'examiner comment les retours d'expériences sur les incidents font progresser la science, l'efficacité des dispositifs et la formation des techniciens ou, à l'inverse, font ralentir le rythme des innovations en instaurant doutes, réticences ou

4. Sur les causes des accidents, cf. Catino, 2002.

5. Salvadori, Levi, 2009 , p. 7. 
pause dans le développement d'une technologie. Dans ce dernier cas, la panne recouvre son sens premier, celui de la navigation, quand les voiles sont orientées pour ne plus avancer, et signifie donc un arrêt prémédité du fonctionnement d'un dispositif technique ou d'une machine. Cette thématique est l'objet de l'article de Philippe Bruyerre, à travers l'étude des " démonstrateurs " d'aérogénérateurs d'électricité, technologies testées en condition réelle d'utilisation et vraie grandeur par EDF avant leur mise sur le marché entre 1958 et 1963 . La mise en sommeil du fonctionnement de ces éoliennes à partir de 1964 est ici présentée comme une décision humaine et sociale, l'accumulation d'incidents ayant débouché sur une mise en panne volontaire provoquée par la perte du soutien social qui était à l'origine de leur mise en œuvre.

\section{Des indices pour une histoire sociale des techniques}

Les pannes et les accidents permettent aussi de positionner l'histoire des techniques sur un champ plus social, d'éclairer une histoire sociale des techniques en mettant en lumière les interactions entre les protagonistes et les dispositifs, et/ou en constituant la porte d'entrée d'une lecture fine des relations de travail, avec la définition des chaînes hiérarchiques et des responsabilités sur les machines et les outils, sur les tâches à accomplir par les acteurs impliqués dans la production de biens et de services. Les incidents mettent au jour les structures et les rapports de pouvoir à l'œuvre dans les collectifs en charge du fonctionnement des machines et des dispositifs techniques. C'est ce que s'attachent à montrer Xavier Daumalin et Olivier Raveux avec l'explosion du navire l'Industrie dans le port le Marseille en 1851 autour de la figure du mécanicien de la marine commerciale. Cette recherche retrace et explique l'évolution des statuts du responsable des machines dans la navigation lors des premières décennies de l'introduction de la vapeur, notamment ses positions sur le navire et dans les textes réglementaires, deux éléments placés au cœur de l'affaire provoquée par l'accident et dont les tribunaux se sont emparés pour rendre la justice civile. Le monde des caissons foncés à l'air comprimé, décrit par Guy Lambert, renvoie à un autre type de coexistence de métiers dans un procédé technique. Occupés à charger ces caissons métalliques en surface pour assurer une contre-pression et participer à leur enfoncement dans le sol, 
les maçons ne descendent ordinairement pas sous terre dans la chambre de travail. Sans être à l'abri des accidents qui guettent tous les ouvriers du bâtiment, à commencer par les chutes, ils ne sont toutefois pas immergés dans un environnement de travail aussi incertain que celui des " tubistes", ainsi que se désignent ceux qui œuvrent dans l'air comprimé. Mesurable au nombre d'accidents survenus parmi ces derniers mais également à la quantité d'études médicales et scientifiques qui leur sont consacrées, les risques encourus éclairent la capacité de cette catégorie professionnelle à incarner la figure phare de ce type de technique constructive, grève à l'appui le cas échéant. Comme le montre également François Jarrige, la mécanisation du battage remodèle non seulement le profil attendu du travailleur agricole mais également la répartition des responsabilités, entre ces derniers et les entrepreneurs possédant ces équipements.

Envisagée sous l'angle des pannes et des accidents, la banalisation des objets techniques dans l'espace quotidien soulève la question des modalités d'usage des dispositifs matériels et des machines, comme le montre l'analyse de Manuel Charpy sur l'utilisation et les utilisateurs des ascenseurs dans le Paris de la Belle Époque. Ici, les techniques sont perçues comme parties prenantes du quotidien et des relations sociales ordinaires. Mais c'est également lorsque les dommages touchent les usagers et non plus seulement des ouvriers, des techniciens ou les opérateurs chargés du fonctionnement des machines que les accidents prennent une toute autre ampleur juridique. Le cas des catastrophes ferroviaires peut en témoigner ${ }^{6}$, mais Xavier Daumalin et Olivier Raveux soulignent également qu’à la différence des accidents survenus précédemment sur des navires de la compagnie Valéry, la présence d'un passager parmi les blessés de l'Industrie est un élément clé pour envoyer l'affaire devant les tribunaux civils.

\section{De la conscience des faits à la prévention des risques}

Le caractère bouleversant voire spectaculaire de l'accident, plus encore que celui de la panne - à moins qu'elle soit d'une ampleur exceptionnelle ${ }^{7}-$,

6. Aldrich, 2006.

7. Il est possible de citer par exemple les célèbres pannes géantes qui occasionnellement ont privé New York ou le Nord-Est des États-Unis d'électricité pendant plus d'une dizaine d'heures. Voir 
détermine leur potentiel médiatique et leur capacité à frapper les esprits ${ }^{8}$. Pour autant, sans doute convient-il ici de distinguer le fait divers de l'événement, tant la nature hors du commun du second - qui « se croit unique »- paraît se démarquer de la tendance à la répétition du premier? Les sondages opérés par François Jarrige dans la presse font apparaître la récurrence des faits constatés. Si les informations fournies par les articles permettent d'établir leur mise en série, tels les cas d'explosion de batteuse à vapeur ou de membres arrachés après avoir été happés dans la transmission des machines, dans quelle mesure les parentés que les lecteurs peuvent relever entre les récits eux-mêmes témoignent-elles d'une forme de narration stéréotypée, trahissant davantage les usages des chroniqueurs ou des pigistes qu'elle ne retranscrit la réalité dans sa complexité ? L'horreur des drames humains s'accordant au registre du fait divers, ce dernier trouve dans les accidents de quoi susciter l'émoi des lecteurs. Manuel Charpy le pointe plus nettement encore à travers le traitement par les journaux illustrés des accidents d'ascenseurs, où l'image renforce la dramatisation.

Si la propension de la presse à l'emphase peut à l'occasion se doubler d'une dénonciation implicite ou soutenir une demande sociale de mesures de sécurité $^{10}$, cette attitude mérite d'être confrontée à un autre ordre du discours plus porté à relativiser les faits. Comme le souligne Philippe Bruyerre à propos des aérogénérateurs, la terminologie convoquée pour désigner ou qualifier les divers aléas techniques trahit nettement le regard porté sur les équipements eux-mêmes, et la volonté de les encourager ou au contraire de les déprécier. Il n’est pas anodin que les "incidents » techniques qui se sont produits dans la mise en ouvre des démonstrateurs se trouvent a posteriori qualifiés d' "accident " lorsqu'il est décidé d'abandonner leur développement. Dans l'échelle des faits, la distinction renvoie certes à une gradation des dommages et des coûts, mais il semble ici que par-delà l'évaluation des faits eux-mêmes - allant des fuites dans le circuit d'huile et de " jeux » dans le système d'orientation de la machine face au vent jusqu'à la rupture d'une pale -, les mots soutiennent le projet politique, le discours des opposants à cette dernière technologie s'écartant résolument de

Nye, 2010, qui étend son analyse aux " pannes " volontaires, motivée par exemple par des raisons militaires.

8. Ambroise-Rendu, 2004.

9. Pour reprendre ici une opposition proposée par Fernand Braudel, 1979, p. 13.

10. Stemmelen, 1983. 
celui de ses promoteurs, plus enclins peut-être à minorer les défaillances techniques qui s'accumulent, par l'emploi d'un vocabulaire mesuré. Sans s'apparenter totalement à de tels artifices du langage, la distance entre arguments commerciaux et réalité matérielle concernant les datacenters est toutefois comparable dès lors que le discours argue de la fiabilité et de la sécurité des dispositifs. En témoigne tout d'abord le profil des " techniciens infrastructures " dont il est attendu, en plus de leurs multiples compétences techniques, qu'ils sachent répondre sereinement aux appels téléphoniques des clients alarmés par toute interruption de service. Mais comme le démontrent Guillaume Carnino et Clément Marquet, l'existence même d'une " armée de petites mains " indispensable au fonctionnement de ces installations entre en contradiction avec un discours enclin à attribuer les pannes et les accidents à une cause humaine - erreur ou négligence - et donc à prôner l'automatisation totale comme remède. L'argument est certes bien ancré dans la civilisation industrielle puisque dès le début du XIX ${ }^{e}$ siècle quels que soient les accidents que causent ou subissent les machines, patrons, experts et hygiénistes sont moins disposés à mettre en question la technique qu'à invoquer la faute ou l'imprudence des travailleurs ${ }^{11}$.

Si l'existence de liens entre les accidents et l'émergence de textes officiels destinés à réguler les risques semble aujourd'hui aller de soi, elle mérite souvent d'être interrogée ${ }^{12}$. Il convient surtout de saisir les épisodes et les facteurs susceptibles d'intervenir dans un tel processus, récurrence des faits, médiatisation, actions en justice. De ce point de vue, il est intéressant de relever, avec Xavier Daumalin et Olivier Raveux, que le jugement rendu par le tribunal civil de Marseille, concernant les statuts hiérarchiques respectifs du capitaine et du mécanicien sur les navires, relève d'une erreur d'appréciation des textes officiels mais préfigure une doctrine que la législation confirme quelques années plus tard. Cet exemple révèle à quel point les études sur les pannes et les accidents peuvent aider à placer les techniques au cœur du juridique et de l'administratif, en occasionnant une réflexion et parfois une redéfinition des normes et des règles. Ces épisodes permettent ainsi d'étudier les contours de l'expertise et des organismes de contrôle publics ou privés en ces domaines.

11. Fressoz, 2014.

12. Le Roux, 2011. 
De nombreuses autres thématiques mériteraient d'être traitées, par exemple dans le domaine économique. Ainsi, les pannes et les accidents permettent d'interroger la performance économique des entreprises, en examinant notamment leurs conséquences financières et leurs coûts en matière de réputation. Il y a là un domaine de recherche reliant l'économique et les techniques demandant encore à être exploré par les historiens. Plus largement, les pannes et les accidents se positionnent sur des terrains multiples, depuis la question des seuils de tolérance des entreprises ou des consommateurs face à ces défaillances ou incidents jusqu’à leurs conséquences dans les domaines juridiques (pénal, civil et commercial). Pour partiel que soit l'ensemble d'articles réunis ici, les coordonnateurs de ce dossier tiennent à souligner le caractère exploratoire de cette entreprise et espèrent que les réflexions présentées avec ces quelques contributions participent d'une dynamique collective de recherche portée par les historiens autour de cette thématique des pannes et des accidents.

\section{Bibliographie}

Aldrich Mark, Death Rode the Rails. American Railroad Accidents and Safety, 1828-1965, Johns Hopkins University Press, Baltimore, 2006.

Ambroise-Rendu Anne-Claude, Petits récits des désordres ordinaires. Les faits divers dans la presse française de la III République à la Grande Guerre, Seli Arslan, Paris, 2004.

Braudel Fernand, Civilisation matérielle, économie et capitalisme XV'-XVIII siècle, vol. 1, Les structures du quotidien, le possible et l'impossible, Armand Colin, Paris, 1979.

Bronstein Jamie, Caught in the Machinery: Workplace Accidents and Injured Workers in the Nineteenth Century, Stanford University Press, Stanford, 2008.

Catino Maurizio, Da Chernobyl a Linate: incidenti tecnologici o errori organizzativi, Carocci, Rome, 2002.

Charpy Manuel, Jarrige François, "Penser le quotidien des techniques. Pratiques sociales, ordres et désordres techniques au XIx siècle", Revue d'histoire $d u$ XIX siècle, $\mathrm{n}^{\circ}$ 45, 2012, p. 7-32.

Chynoweth John C., Accident Prone: a History of Technology, Psychology and Misfits of the Machine Age, University of Chicago Press, Chicago, 2009.

Fressoz Jean-Baptiste, «L'émergence de la norme technique de sécurité en France vers 1820 ", Le Mouvement social, n² 249, 2014, p. 73-89.

Fressoz Jean-Baptiste, L'Apocalypse joyeuse. Une histoire du risque technologique, Le Seuil, Paris, 2012. 
Landes David, L'Europe technicienne ou le Prométhée libéré. Révolution technique et libre essor en Europe occidentale de 1750 à nos jours, Gallimard, Paris, 1975 [Cambridge University Press, Cambridge, 1969].

Le Roux Thomas (dir.), Risques industriels : savoirs, régulations, politiques d'assistance : fin XVII'-début XXe siècle, PUR, Rennes, 2016.

Le Roux Thomas, "Accidents industriels et régulation des risques : l'explosion de la poudrerie de Grenelle en 1794 ", Revue d'histoire moderne et contemporaine, n $58-6,2011$, p. 36-62.

Lekéal Farid, Dubois Bruno, Crochepeyre Nathalie (dir.), « La réparation des accidents du travail : pratiques et acteurs, $\mathrm{XIX}^{\mathrm{e}}-\mathrm{XX}^{\mathrm{e}}$ siècles, France, Belgique ", Revue du Nord, hors-série n 34, 2016.

Nye David E., When the Lights Went Out. A History of Blackouts in America, MIT Press, Cambridge (Mass.), 2010.

Salvadori Mario, Levi Matthys, Pourquoi ça tombe ?, Éditions Parenthèses, Marseille, 2009 [W.W. Norton \& Compagny inc., New York, 1992, édition augmentée en 2002].

Stemmelen Hélène, "Une catastrophe technologique au XIx ${ }^{e}$ siècle à travers le journal Le Temps», Culture technique, $\mathrm{n}^{\circ} 11,1983$, p. 308-315. 